\title{
Jet outflow and gamma-ray emission correlations in S5 0716+714 ${ }^{\star}$
}

\author{
B. Rani ${ }^{1}$, T. P. Krichbaum ${ }^{1}$, A. P. Marscher ${ }^{2}$, S. G. Jorstad ${ }^{2}$, J. A. Hodgson ${ }^{1}$, L. Fuhrmann ${ }^{1}$, and J. A. Zensus ${ }^{1}$ \\ 1 Max-Planck-Institut für Radioastronomie (MPIfR), Auf dem Hügel 69, 53121 Bonn, Germany \\ e-mail: brani@mpifr-bonn.mpg.de \\ 2 Institute for Astrophysical Research, Boston University, 725 Commonwealth Avenue, Boston, MA 02215, USA \\ Received 12 August 2014 / Accepted 30 September 2014
}

ABSTRACT

\begin{abstract}
Using millimeter very long baseline interferometry (VLBI) observations of the BL Lac object S5 0716+714 from August 2008 to September 2013, we investigate variations in the core flux density and orientation of the sub-parsec scale jet, i.e. position angle. The $\gamma$-ray data obtained by the Fermi Large Area Telescope are used to investigate the high-energy flux variations over the same time period. For the first time in any blazar, we report a significant correlation between the $\gamma$-ray flux variations and the position angle variations in the VLBI jet. The cross-correlation analysis also indicates a positive correlation such that the mm-VLBI core flux density variations are delayed with respect to the $\gamma$-ray flux by $82 \pm 32$ days. This suggests that the high-energy emission is coming from a region located $\geq(3.8 \pm 1.9)$ parsecs upstream of the mm-VLBI core (closer to the central black hole). These results imply that the observed inner jet morphology has a strong connection with the observed $\gamma$-ray flares.
\end{abstract}

Key words. galaxies: active - BL Lacertae objects: individual: S5 0716+714 - galaxies: jets - gamma rays: galaxies radio continuum: galaxies

\section{Introduction}

The origin of high-energy emission has long been a key question in active galactic nuclei (AGN) physics. A combination of high-resolution very long baseline interferometry (VLBI) images with broadband flux variability measurements is a unique way to probe the emission mechanisms at the bases of jets in a subclass of AGN called blazars, one of whose jets points toward the observer's line of sight. High-resolution mm-VLBI observations offer a unique possibility of studying the structural evolution in the inner parsecs of jets, which are proposed as the sites of the high-energy emission region (e.g., Lähteenmäki \& Valtaoja 2003; Rani et al. 2013c,b; Marscher et al. 2008; Schinzel et al. 2012; Fuhrmann et al. 2014, and references therein).

Non-radial motion, helical paths of the jet features, curved jet structures, and variations in the direction of the inner jet flow, i.e. position angle or jet wobbling, have often been observed in blazars (Britzen et al. 2009; Bach et al. 2005; Rastorgueva et al. 2011; Lister et al. 2013; Molina et al. 2014). In some cases the inner jet position angle (PA) variations were found to correlate with the flux density variations at radio frequencies (Britzen et al. 2009; Liu et al. 2012) and also with X-rays (Chatterjee et al. 2008). However, a correlation between $\gamma$-ray flux and PA variations has not been reported so far.

In this paper, we report a correlation between $\gamma$-ray flux variations with the VLBI core flux density and the direction of the inner jet flow in the BL Lac object S5 0716+714. The mmVLBI observations over the past five years were used to investigate the correlation. The aim of the study is to provide better constraints on the location of the high-energy emission region with an emphasis on the inner jet region kinematics and its correlation with the high-energy flux emission. A detailed analysis of the complete jet kinematics will follow in a subsequent paper.

\footnotetext{
* Appendix $\mathrm{A}$ is available in electronic form at http://www . aanda.org
}

The BL Lac object S5 $0716+714$ is one of the most intensively studied blazars because of its extreme variability properties across the whole electromagnetic spectrum (Villata et al. 2008; Fuhrmann et al. 2008; Rani et al. 2013a,b; Larionov et al. 2013, and references therein). The source has a featureless optical continuum with the redshift roughly constrained to the range $0.2315<z<0.3407$ (Danforth et al. 2013); here we used $z \approx 0.31$ (Nilsson et al. 2008). VLBI studies of the source show a core-dominated jet pointing toward the north (Bach et al. 2005; Britzen et al. 2009), and Very Large Array (VLA) observations show a halo-like jet misaligned with it by $\sim 90^{\circ}$ (Antonucci et al. 1986). The broadband flaring behavior of the source is even more complex. The observed flux density light curves very often reflect rapid flaring activity superimposed on top of a broad and slow variability trend (Rani et al. 2013a; Raiteri et al. 2003).

\section{Observations and data reduction}

\subsection{Very long baseline interferometry}

For the jet kinematics study, we used the mm-VLBI data of the source observed between August 2008 and September 2013. The $7 \mathrm{~mm}$ (43 GHz) data were a result of the Boston University group ${ }^{1}$ monthly monitoring program with the Very Long Baseline Array (VLBA). The $3 \mathrm{~mm}(86 \mathrm{GHz})$ observations were performed using the Global mm-VLBI Array $\left(\mathrm{GMVA}^{2}\right)$. In total, we had observations at 63 epochs over the past 5 years. Data reduction was performed using the standard tasks of the Astronomical Image Processing System (AIPS) and Difmap (Shepherd et al. 1994). Imaging of the source (including amplitude and phase self-calibration) was performed using the CLEAN algorithm (Högbom 1974) and SELFCAL procedures in the Difmap package (Shepherd et al. 1994). Further details of the data reduction can be found in Jorstad et al. (2005).

\footnotetext{
1 http://www.bu.edu/blazars

2 http://www3.mpifr-bonn.mpg.de/div/vlbi/globalmm/ index.html
} 
We modeled the observed brightness distribution of the radio emission by multiple circular Gaussian components providing positions, flux densities, and sizes of the distinct bright features in the jet using the Difmap package. For all model fits, we used the brightest component as a reference and fixed its position to $(0,0)$. The final number of jet components necessary to fit the data adequately was reached when adding an extra component did not lead to a significant improvement in the fit. Uncertainties of the parameter fits were estimated following Krichbaum et al. (1998) and Jorstad et al. (2005).

\subsection{Gamma rays}

We employed here the $100 \mathrm{MeV}-300 \mathrm{GeV}$ data of the source from August 4, 2008 to September 30, 2013, which were observed in survey mode by the Fermi Large Area Telescope (LAT, Atwood et al. 2009). We analyzed the LAT data using the standard ScienceTools (software version v9.32.5) and instrument response function P7REP_SOURCE. Photons in the source event class were selected for the analysis. Because, the VLBI observations of the source have an average sampling of about one month, we preferred a time binning of one month for the $\gamma$-ray photon flux light curves. We obtained similar results for weekly binned light curves. The monthly binned light curves of the source at $E>100 \mathrm{MeV}$ were produced by modeling the spectra over each bin by a simple power law $\left(N(E)=N_{0} E^{-\Gamma}, N_{0}\right.$ : prefactor, and $\Gamma$ : power law index). For this analysis, we used the unbinned maximum-likelihood algorithm (Mattox et al. 1996). The analysis performed in this paper is very similar to what is reported in Rani et al. (2013a), to which we refer for details.

\section{Analysis and results}

\subsection{Jet orientation variations}

To determine the inner jet orientation, we used an annular region up to 0.2 mas from the core at $(0,0)$. We fitted a straight line between the core and its adjacent component (see Fig. A.1). The PA of this line provided a reasonably good estimate of the direction of the inner portion of the jet. However, it should be noted that the derived PA values could be influenced by choices made for the fitted Gaussians (e.g. elliptical vs. circular) and the total number of Gaussian components in the inner jet region. We therefore explored an independent approach to calculate the inner jet PA. We determined the inner jet PA at each epoch by taking a flux density-weighted PA average of all the clean delta components $^{3}$ three times above the image noise level in the annular region (see Fig. A.1). We used the $1 \sigma$ distribution of the clean delta components around the mean PA axis as uncertainties. The two approaches suggest consistent PA variations as shown in Fig. 1a.

\subsection{Gamma-ray flux vs. PA variations}

To compare the $\gamma$-ray flux variations with the PA variations, we plot the two on top of each other (see Fig. 2a). For a visual comparison, we divide the whole period into six segments, T1 to T6. The inner-jet PA and $\gamma$-ray flux appear to vary together during $\mathrm{T} 1$ and T2. After this, the variations diverge until T6, when they are again similar. This suggests a relation between the two properties of the blazar that is sometimes strong and at other times complex or non-existent.

\footnotetext{
3 The clean delta components represent the deconvolved brightness distribution of the source structure.
}

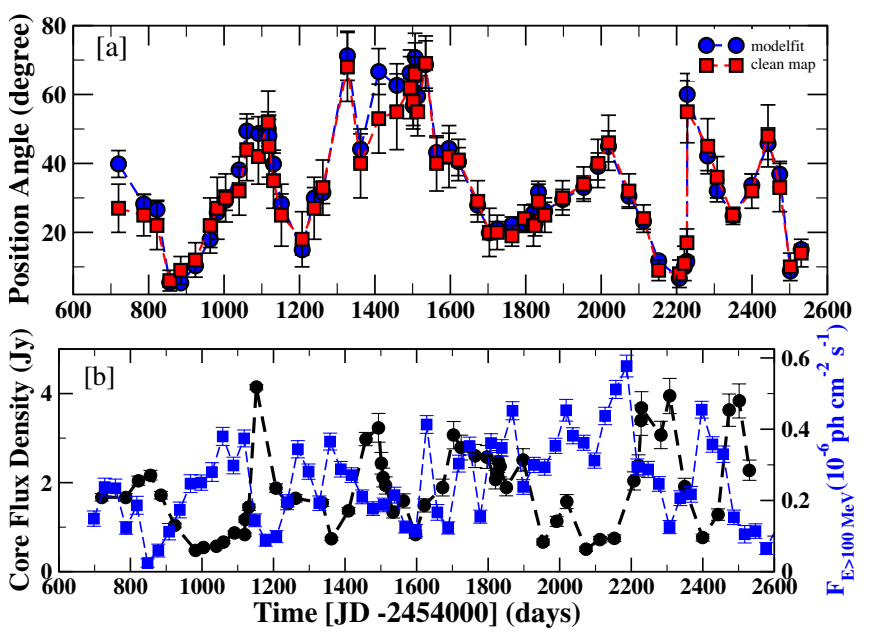

Fig. 1. a) Position angle (PA) variations in the central region of the jet. The blue circles show the PA calculated using model-fitting, while those estimated directly from clean maps are in red (square symbols). b) mmVLBI core flux density variations (black circles) over the same time period superimposed with the monthly averaged $\gamma$-ray flux variations (blue squares).
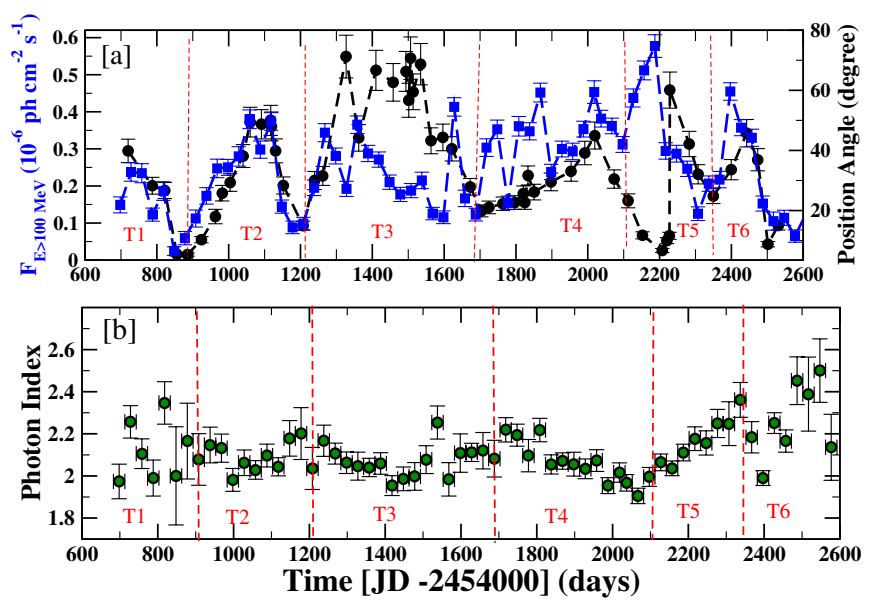

Fig. 2. a) Monthly averaged $\gamma$-ray flux light curve (blue squares) superimposed on the model-fitted PA curve (black circles). b) Gamma-ray photon index versus time.

To quantify the apparent correlation of the PA and $\gamma$-ray flux variations, we employed the discrete cross-correlation function (DCF) analysis method (Edelson \& Krolik 1988). Figure 3a shows the DCF analysis results of the monthly averaged $\gamma$-ray flux versus the inner jet PA. The DCF curve shows a prominent peak at $47 \pm 22$ days. The given uncertainty in the time lag value here and in the following sections is the half-width at the $90 \%$ point of the best-fit Gaussian function to the DCF curve. The significance of the DCF analysis was tested using simulations as discussed in Appendix A, and the 95\%, 99\%, and 99.97\% confidence levels are shown in Fig. 3a. The simulations imply that the significance of the measured correlation is $>3 \sigma$. It is important to note that the PA values are measured by fixing the core position to $(0,0)$ within a region up to 0.2 mas; consequently, the observed PA variations can either be related to the mm-VLBI core or the jet flow farther downstream of the core, or both. The observed time lag therefore cannot be used for physical calculations, e.g., to estimate the distance between the two emission regions, because the reference/zero point of the PA measurements is arbitrary. However, what is more important is that the $\gamma$-ray flux variations correlate significantly with the PA variations of the inner jet. 

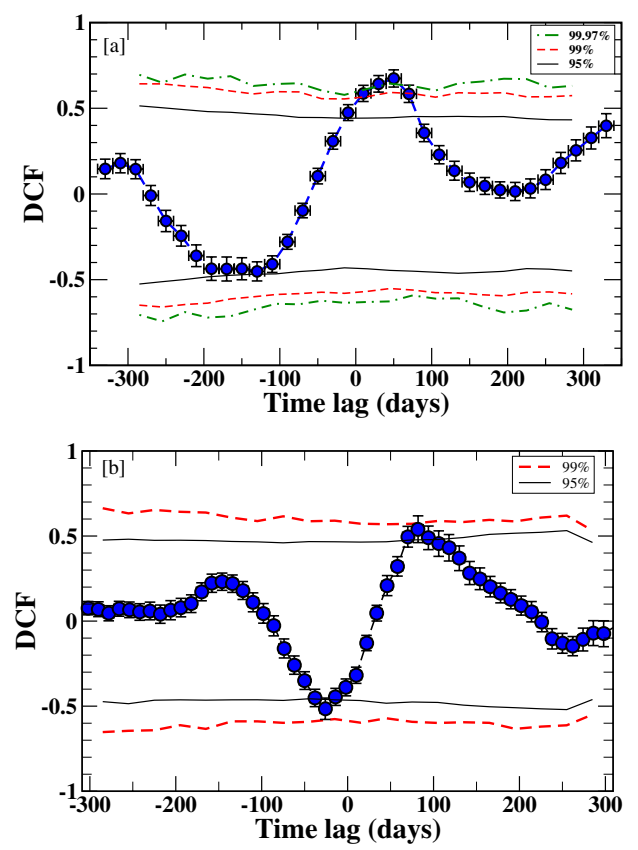

Fig. 3. a) DCF analysis curve of the $\gamma$-ray flux and PA variations in the inner jet region. The lines show confidence levels as discussed in the text. b) DCF analysis curve between the $\gamma$-ray and core flux density variations. A positive time lag implies that $\gamma$-ray flux variations lead core flux density and PA variations.

In Fig. 2b, we plot the $\gamma$-ray photon index $(\Gamma)$ as a function of time. The main deviations from apparently random fluctuations are seen during T4 and T5. During T4, $\Gamma$ drops from $2.19 \pm 0.05$ to $1.90 \pm 0.04$, and later the spectrum softens to a photon index value $=2.36 \pm 0.08$ at the end of T5. Since the photon index variations cannot be interpreted as purely geometrical effects, this suggests that the flux variations are related to changes in both the physical conditions in the plasma and Doppler beaming.

\subsection{Gamma-ray flux vs. core flux density variations}

Figure $1 \mathrm{~b}$ shows the monthly binned $\gamma$-ray flux light curve plotted on top of the mm-VLBI core flux density ${ }^{4}$ light curve. For several events, the peak of the $\gamma$-ray flare appears to coincide with the onset of the radio flare, and to investigate it, we used the DCF method. The DCF curve (Fig. 3b) shows a peak at $82 \pm 32$ days and a dip at $-(29 \pm 25)$ days. The significance of the cross-correlation was tested via simulations as discussed in Sect. A. In Fig. 3b, the lines show the $95 \%$ and $99 \%$ confidence levels. The simulations, therefore, revealed that the significance of both the correlation (at $82 \pm 32$ days) and the anti-correlation (at $-(29 \pm 25)$ days) is $\sim 99 \%$. An anti-correlation implies that $\gamma$-ray flares lead those at radio wavelengths or vice-versa such that the maximum of one coincides with the minimum of other. We found that the radio jet flux density further downstream of the core is very faint and does not show any correlated variation with the $\gamma$-ray light curve, suggesting that the $\gamma$-ray flares are not produced downstream of the core. This implies that $\gamma$ rays are produced upstream of the core, which is also supported by the positive correlation between $\gamma$-ray and core flux density light curves with the former leading the latter by $82 \pm 32$ days. We note that for a larger sample of $\gamma$-ray blazars, similar results

\footnotetext{
4 We used a mean value of the measured optically-thin spectral index, $\alpha_{\text {thin }}=0.4$ (Rani et al. 2013a) to scale the $3 \mathrm{~mm}$ flux density measurements to those at $7 \mathrm{~mm}$.
}
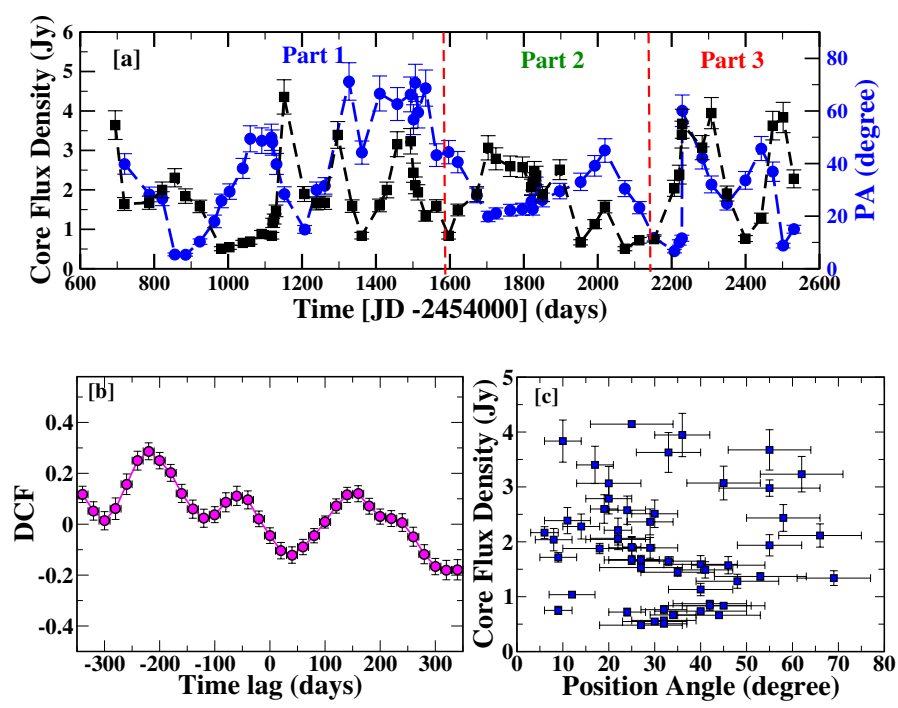

Fig. 4. a) Core flux density light curve superimposed on the PA curve. b) The DCF analysis curve of the core flux density vs. PA variations. c) core flux density vs. PA plot.

( $\gamma$-ray leading mm-radio flares) have recently been reported by Fuhrmann et al. (2014).

\subsection{Core flux density vs. PA variations}

A comparison of the observed core flux density and PA variations is shown in Fig. 4a. For a visual comparison, we divide the whole period into three segments, Parts 1 to 3 . The core flux density and PA appear to vary together for Part 3, while the variations diverge during Parts 1 and 2. The formal DCF analysis of all data does not reveal a significant correlation between the two (see Fig. 4b). However, the absence of a significant correlation does not rule out weak correlations or much more complicated behavior. The core flux density vs. PA plot (Fig. 4c) shows a ring-like pattern with its center at $\sim 40^{\circ}$, which suggests some sort of correlation between the two.

\section{Discussion and conclusion}

Our analysis suggests a strong correlation between high-energy emission and inner jet morphology. We found a strong correlation between $\gamma$-ray flux variations and PA variations. The observed time lag of $82 \pm 32$ days between $\gamma$-ray and core flux density variations places the $\gamma$-ray emission region upstream of the $m m-V L B I$ core by $\geq(3.8 \pm 1.9)$ parsec (deprojected using a viewing angle, $\theta \leq 4.9^{\circ}$ and apparent jet speed $\beta_{\text {apparent }}=10$, Bach et al. 2005). These correlations indicate that some change in the jet structure first triggers a $\gamma$-ray flare, and later the event has traveled 0.2 mas down the jet, so that either the jet PA between the core and the first jet component changes, or at that point the event has just traveled to the core and caused the core to shift in the transverse direction. In a simple scenario, we would also expect a correlation between core flux density and PA variations in the optically-thin case; however, the correlation could be weaker for a partially optically-thick core.

Systematic variations in the orientation of parsec to subparsec scale VLBI jets have been observed in many sources (e.g., Bach et al. 2005; Lister et al. 2013; Molina et al. 2014, and references therein). The exact origin of these variations is not clear yet, although accretion disk precession, orbital motion of the accretion system, and instabilities (Magnetohydrodynamic (MHD), and Kelvin-Helmholtz (KH)) in the jet flow have all 
A\&A 571, L2 (2014)

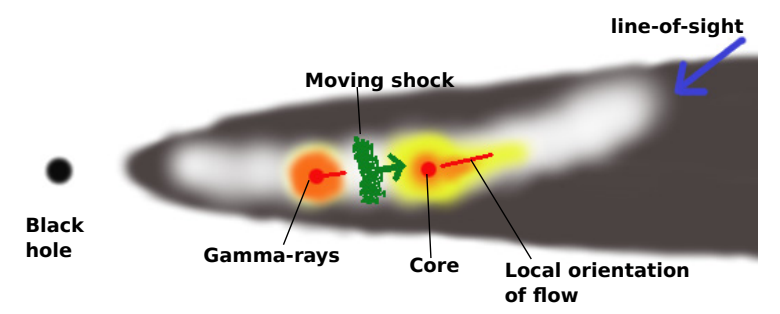

[a]

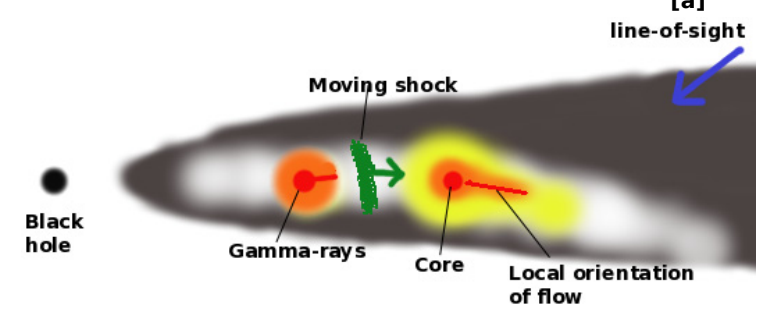

Fig. 5. Sketch for the proposed scenario in the BL Lac S5 0716+714 (not to scale). The high-density/pressure regions, shown in light gray (superimposed on the underlying jet flow, which is in dark), brighten relative to other regions of the jet by the passage of a moving shock. a) Case for a strong correlation; and b) a weak correlation.

been suggested. The relatively short variability time scales ( 200 days) involved and the observed non-ballistic jet motion in S5 0716+714 (Bach et al. 2005; Britzen et al. 2009) most likely exclude geometric precession due to a binary black hole. In relativistic jet models (Blandford \& Payne 1982; Blandford \& Znajek 1977), spatially bent jets and helical fluid patterns are a natural consequence of KH-instabilities (Hardee \& Eilek 2011) or MHD instabilities, or both (Meier et al. 2001). In such "magnetic jets", the helical structure is formed by a twist of the magnetic filaments through and around the conical jet, which may explain the observed PA variations in the source.

In a tentative model (shown in Fig. 5), the observed $\gamma$-ray flux and PA variations can be interpreted as a moving shock propagating down a relativistic jet with non-axisymmetric pressure or density gradients/patterns, or a shock moving in a bent jet. A moving shock will induce significantly increased emission at the locations where it intersects with regions of enhanced electron density or magnetic field. The measured correlations suggest that the $\gamma$-ray flares precede the mm-VLBI core flares, and the time lag depends on the physical conditions of the emission region. Longer time lags can be expected because of opacity effects or if the two emission regions are separated (as shown in Fig. 5). Because Doppler boosting is a sensitive function of the viewing angle, substantial changes in amplitude of jet emission can be seen by the observer. Correlated variation between the $\gamma$-ray emission and orientation of the jet flow is obvious if the two share the same boosting cone as shown in Fig. 5a; however, if the two emission regions are pointed in different directions, the correlation between $\gamma$-ray flux and PA would be weaker (Fig. 5b). Therefore, this scenario successfully explains why sometimes we see very strong correlations and sometimes not (see Fig. 2). One could also consider instability patterns moving downstream and passing the two emission regions at different angles, or even a rotation of the (helical) jet around its own $z$-axis. All models would cause very similar variations of the viewing angle, which is responsible for the observed correlation between $\gamma$-ray flux and apparent jet PA. A correlated variation between the core flux density and the PA curves is expected in a simple scenario. The expected correlation is, however, not detected with the current observations.

The observed correlation between the $\gamma$-ray flux variations and the inner jet kinematics are a challenge for the available relativistic jet models. Sub-mm (3 mm/1 mm) VLBI monitoring with denser time sampling would be required to better understand the parsec scale jet morphology. In addition to this, the aforementioned hypotheses need to be developed with magnetohydrodynamic simulations to better understand the jet-launching region.

Acknowledgements. The Fermi-LAT Collaboration acknowledges support from a number of agencies and institutes for both development and the operation of the LAT as well as scientific data analysis. These include NASA and DOE in the United States, CEA/Irfu and IN2P3/CNRS in France, ASI and INFN in Italy, MEXT, KEK, and JAXA in Japan, and the K. A. Wallenberg Foundation, the Swedish Research Council and the National Space Board in Sweden. Additional support from INAF in Italy and CNES in France for science analysis during the operations phase is also gratefully acknowledged. This study makes use of $43 \mathrm{GHz}$ VLBA data from the VLBA-BU Blazar Monitoring Program (VLBABU-BLAZAR; http://www.bu.edu/blazars/VLBAproject.html), funded by NASA through the Fermi Guest Investigator Program. The VLBA is an instrument of the National Radio Astronomy Observatory. The National Radio Astronomy Observatory is a facility of the National Science Foundation operated by Associated Universities, Inc. We would like to thank Chuck Dermer, the internal referee for the Fermi-LAT Collaboration, Eric C. Brown, Andrei Lobanov, Jeremy Perkins, and David Thompson for their useful suggestions and comments. B.R. is greatful to Moritz Boeck, S. Vaughan, D. Emmanoulopoulos, and I. McHardy for the useful discussion of calculating the significance of correlations. We thank the referee for constructive comments.

\section{References}

Antonucci, R. R. J., Hickson, P., Olszewski, E. W., \& Miller, J. S. 1986, AJ, 92, 1 Atwood, W. B., Abdo, A. A., Ackermann, M., et al. 2009, ApJ, 697, 1071

Bach, U., Krichbaum, T. P., Ros, E., et al. 2005, A\&A, 433, 815

Blandford, R. D., \& Payne, D. G. 1982, MNRAS, 199, 883

Blandford, R. D., \& Znajek, R. L. 1977, MNRAS, 179, 433

Britzen, S., Kam, V. A., Witzel, A., et al. 2009, A\&A, 508, 1205

Chatterjee, R., Jorstad, S. G., Marscher, A. P., et al. 2008, ApJ, 689, 79

Danforth, C. W., Nalewajko, K., France, K., \& Keeney, B. A. 2013, ApJ, 764, 57

Edelson, R. A., \& Krolik, J. H. 1988, ApJ, 333, 646

Emmanoulopoulos, D., McHardy, I. M., \& Papadakis, I. E. 2013, MNRAS, 433, 907

Fuhrmann, L., Krichbaum, T. P., Witzel, A., et al. 2008, A\&A, 490, 1019

Fuhrmann, L., Larsson, S., Chiang, J., et al. 2014, MNRAS, 441, 1899

Hardee, P. E., \& Eilek, J. A. 2011, ApJ, 735, 61

Högbom, J. A. 1974, A\&AS, 15, 417

Jorstad, S. G., Marscher, A. P., Lister, M. L., et al. 2005, AJ, 130, 1418

Krichbaum, T. P., Alef, W., Witzel, A., et al. 1998, A\&A, 329, 873

Lähteenmäki, A., \& Valtaoja, E. 2003, ApJ, 590, 95

Larionov, V. M., Jorstad, S. G., Marscher, A. P., et al. 2013, ApJ, 768, 40

Lister, M. L., Aller, M. F., Aller, H. D., et al. 2013, AJ, 146, 120

Liu, X., Mi, L., Liu, B., \& Li, Q. 2012, Ap\&SS, 342, 465

Marscher, A. P., Jorstad, S. G., D’Arcangelo, F. D., et al. 2008, Nature, 452, 966

Mattox, J. R., Bertsch, D. L., Chiang, J., et al. 1996, ApJ, 461, 396

Meier, D. L., Koide, S., \& Uchida, Y. 2001, Science, 291, 84

Molina, S. N., Agudo, I., Gómez, J. L., et al. 2014, A\&A, 566, A26

Nilsson, K., Pursimo, T., Sillanpää, A., Takalo, L. O., \& Lindfors, E. 2008, A\&A, 487, L29

Raiteri, C. M., Villata, M., Tosti, G., et al. 2003, A\&A, 402, 151

Rani, B., Krichbaum, T. P., Fuhrmann, L., et al. 2013a, A\&A, 552, A11

Rani, B., Krichbaum, T. P., Lott, B., Fuhrmann, L., \& Zensus, J. A. 2013b, Adv. Space Res., 51, 2358

Rani, B., Lott, B., Krichbaum, T. P., Fuhrmann, L., \& Zensus, J. A. 2013c, A\&A, 557, A71

Rastorgueva, E. A., Wiik, K. J., Bajkova, A. T., et al. 2011, A\&A, 529, A2

Schinzel, F. K., Lobanov, A. P., Taylor, G. B., et al. 2012, A\&A, 537, A70

Shepherd, M. C., Pearson, T. J., \& Taylor, G. B. 1994, BAAS, 26, 987

Vaughan, S. 2005, A\&A, 431, 391

Villata, M., Raiteri, C. M., Larionov, V. M., et al. 2008, A\&A, 481, L79 


\section{Appendix A: Testing the correlation significance}

The significance of correlations in the DCF analysis was determined using simulations. To do so, we first estimated the PSD (power spectral density) slope of the $\gamma$-ray light curve following Vaughan (2005). The estimated PSD slope for $0716+714$ is $-(0.93 \pm 0.24)$. The next step is to generate the simulated light curves using the PSD slope. One can use the online available IDL code ${ }^{5}$. However, the simulated light curves using this code follow a Gaussian distribution, which is normally not the case for the observed light curves because a majority of them are burst-like events. This has to be taken into account while estimating the correlation significances (see Emmanoulopoulos et al. 2013 , for details). We therefore checked the distribution of the observed $\gamma$-ray light curve and found that the underlying distribution is similar to a Gaussian one. We generated a series of 100000 light curves using the online available IDL code. The next step is to sample the simulated light curves at the same times and bin widths as the observations. We cross-correlated the simulated $\gamma$-ray light curves with the observed PA curve. Finally, we calculated the distribution of the DCF values as a function of time lag. For each time delay, we estimated the 0.025 and 0.975 quantiles corresponding to the upper and lower limits of the $95 \%$ confidence bands. However, these confidence levels are obtained for a single trial; i.e. if we already know the time lag between the two light curves. Since we do not have the time lag information a priori, we always use a search window depending on the duration of observations. To correct for this effect (called the "look elsewhere effect"), one has to include the total number of trials, which is the number of data points in the given search window (for details see Sect. 4.1 in Vaughan 2005). For $\mathrm{N}$ points, the confidence level, $\epsilon$, is $(1-(1-\epsilon) / N) \times 100 \%$; i.e. the $95 \%$ confidence level for 10 data points in a given time lag range should be $[1-(1-0.95) / 10] \times 100=99.5 \%$ confidence level for a single data point.

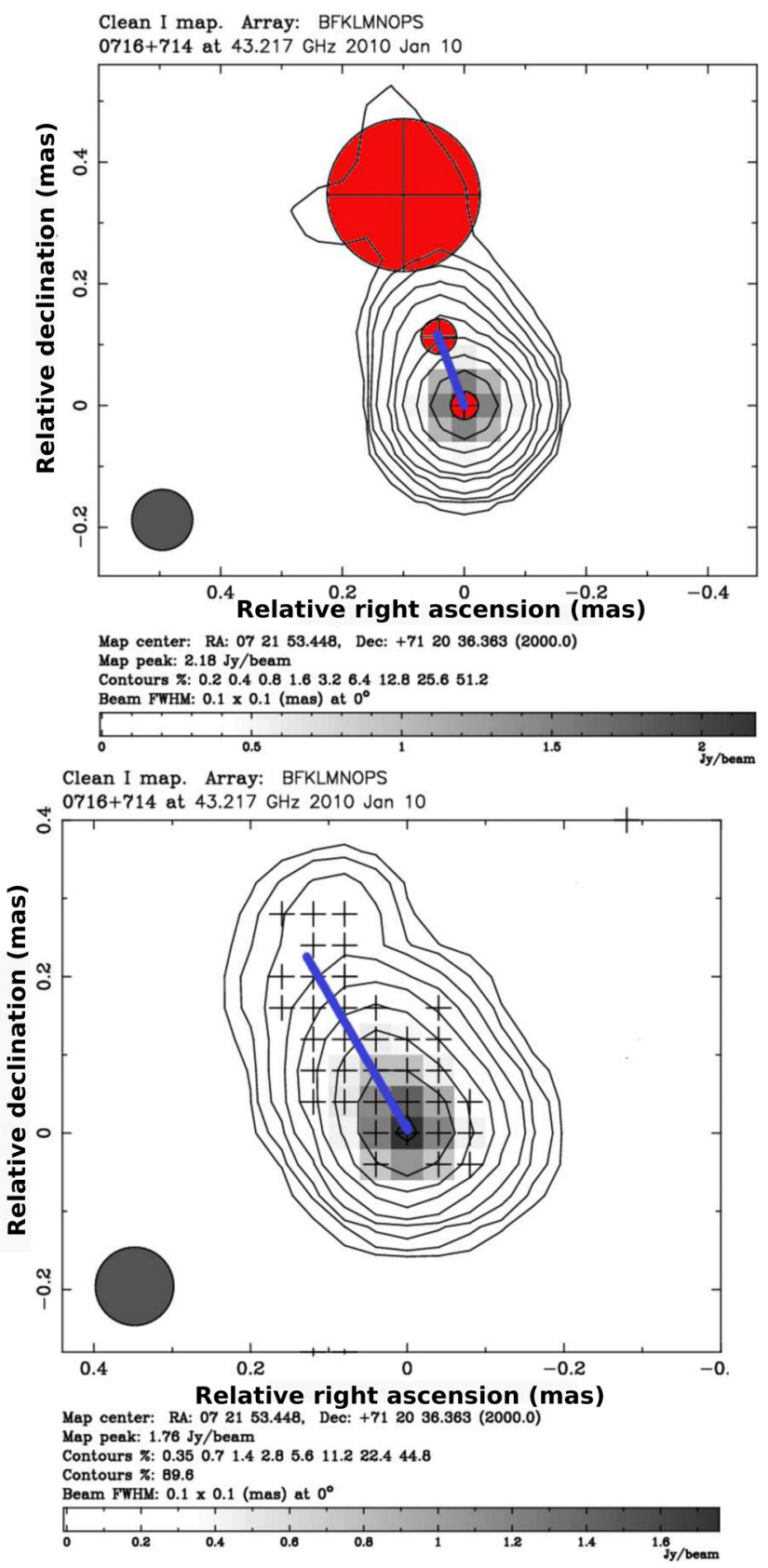

Fig. A.1. Example of $43 \mathrm{GHz}$ VLBI images of S5 0716+714 observed on January 10, 2010 resolved with a beam size of 0.1 mas (gray circle in the bottom left corner of maps). Contour maps are superimposed with Gaussian model-fit components (red circles in the top panel) and clean delta components (plus symbols in the bottom panel). The blue lines mark the inner jet orientation.

\footnotetext{
5 http://astro.uni-tuebingen.de/software/idl/aitlib/ timing/timmerlc.html
} 